\title{
El proceso de enseñanza-aprendizaje de la Historia del Cine a través de una estrategia de storytelling y storydoing teatral aplicada al ámbito universitario
}

The teaching-learning process of the History of Cinema through a strategy of storytelling and theatrical storydoing applied to the university environment

0 processo ensino-aprendizagem da História do Cinema através de uma estratégia de storytelling e storydoing teatral aplicada ao ambiente universitário

\author{
Andrea Castro-Martinez \\ Docente e investigadora \\ (Universidad de Málaga) \\ https://orcid.org/0000-0002-2775-625X \\ España \\ Pablo Diaz-Morilla \\ Director de los BA (Hons) in Communications y de las \\ Enseñanzas artísticas superiores en Diseño de EADE \\ (University of Wales Trinity Saint David) \\ Investigador \\ (Universidad de Málaga) \\ https://orcid.org/0000-0002-7914-3391 \\ España
}

Fecha de recepción: 1 de marzo de 2019

Fecha de revisión: 8 de junio de 2019

Fecha de publicación: 1 de julio de 2019

Para citar este artículo: Castro-Martinez, A. y Diaz-Morilla. P. (2019). El proceso de enseñanza-aprendizaje de la Historia del Cine a través de una estrategia de storytelling y storydoing teatral aplicada al ámbito universitario, Icono 14, 17 (2), 154-181. doi: 10.7195/ri14.v17i2.1378 


\section{Resumen}

Las asignaturas relacionadas con la historia suelen presentar dificultades para el alumnado y en ocasiones resultar tediosas por ser interpretadas como una recopilación de fechas y nombres a veces aparentemente inconexos. Esta investigación analiza una práctica docente alternativa aplicada a la enseñanza-aprendizaje de la historia del cine a nivel universitario con el objetivo de conocer la utilidad del storytelling y storydoing teatral en este ámbito, así como las percepciones del alumnado sobre esta iniciativa que pretende convertirlo en protagonista del aprendizaje sobre cine. Con el objetivo de dinamizar y facilitar el proceso de aprendizaje significativo de la historia del cine se ha desarrollado el proyecto 'El cine a través del storytelling y el teatro' en el marco de la asignatura Realización del tercer curso de los Grados en Comunicación en EADE University of Wales en Málaga. Se ha aplicado una metodología que refuerza el trabajo sobre los aspectos teóricos relacionados con la historia del cine a través de una dinámica de role playing en la que el alumnado interpreta, dirige y realiza audiovisualmente textos teatrales en los que la trama y los personajes se centran en personalidades relevantes para el desarrollo del séptimo arte.

Los resultados indican que esta metodología aplicada a la enseñanza de contenidos sobre cine se ha revelado efectiva para transferir al alumnado conocimientos teóricos propios de la materia de un modo innovador al tiempo que desarrolla habilidades transversales relacionadas con la comunicación no verbal, la comunicación en público, el trabajo colaborativo y la técnica audiovisual.

Palabras clave: Cine; Aprendizaje; Teatro; Storytelling; Role playing; Dramatización

\section{Abstract}

The subjects related to the History usually present difficulties for the students and occasionally they can be tedious to be interpreted as a compilation of dates and names, sometimes when they seem unconnected. This research analyses an alternative teaching practice applied to the teaching-learning of the history of cinema at university level with the aim of finding out the usefulness of storytelling and theatrical storydoing in this field, as well as students' perceptions of this initiative that aims to make them the protagonists of learning about cinema. In order to invigorate 
and facilitate the process of meaningful learning of the history of cinema, the project 'Cinema through storytelling and theatre' has been developed within the framework of the subject Filming of the third year of the Degrees in Communication at EADE University of Wales in Malaga. The methodology that has been applied reinforces the work on the theoretical aspects related to the history of cinema through a dynamics of role playing in which the students interpret, direct and perform audiovisual theatrical texts in which the plot and the characters are centered in personalities relevant to the development of the seventh art. Through a qualitative and quantitative research, the texts used and the students' perceptions about this educational practice that applies storytelling and combines it with storydoing to turn students into protagonists of film learning have been studied.

The results indicate that this methodology applied to the teaching of film content has proved effective in transferring the students' theoretical knowledge of the subject in an innovative way while developing cross-cutting skills related to non-verbal communication, public communication, collaborative work and the audiovisual technique.

Key Words: Cinema; Learning; Theatre; Storytelling; Role playing; Drama

\section{Resumo}

Os assuntos relacionados com a história costuma apresentar dificuldades para os alunos e por vezes ser interpretado como uma compilação com as datas e nomes aparentemente desconexos. Esta pesquisa analisa uma prática pedagógica alternativa aplicada ao ensino-aprendizagem da história do cinema em nível universitário com o objetivo de descobrir a utilidade da narrativa e do storydoing teatral neste campo, bem como as percepções dos alunos sobre esta iniciativa que visa torná-los protagonistas do aprendizado sobre cinema. Com o objetivo de melhorar e facilitar o processo de aprendizagem da história do cinema. Desenvolveu-se o projeto 'Cinema através de storytelling e teatro', no âmbito de curso Filming do terceiro ano do Grau em Comunicação na EADE University of Wales em Málaga. Aplicou-se uma metodologia que reforça o trabalho sobre os aspectos teóricos relacionados com a história do cinema através de uma dinâmica de role playing em que os alunos interpretam, dirigem e executam textos teatrais audiovisuais nos quais 
a trama e os personagens enfocam personalidades relevantes para o desenvolvimento da sétima arte. Por meio de pesquisa qualitativa e quantitativa, os textos utilizados e as percepções dos estudantes sobre essa prática educacional, isso se aplica a storytelling e combina com a storydoing para transformar os alunos em protagonistas da aprendizagem de filmes.

Os resultados indicam que esta metodologia aplicada ao ensino o conteúdo cinematográfico revelou-se eficaz na transferência de conhecimentos teóricos do assunto para os alunos de forma inovadora, desenvolvendo competências transversais relacionadas com a comunicação não-verbal, comunicação pública, trabalho colaborativo e técnica audiovisual.

Palavras chave: Cinema; Aprendizagem; Teatro; Narratividade; Role playing; Dramatização

\section{Introducción}

Las técnicas y metodologías alternativas a la clase magistral son un recurso didáctico muy efectivo para fomentar la comprensión de determinados temas dentro del aula. Las asignaturas relacionadas con la historia suelen tener un cierto hándicap de tediosas entre el alumnado que, a grandes rasgos, suele tener dificultades para retener conceptos como fechas y nombres. Estos son los motivos que llevaron a plantear el uso del storytelling teatral en el marco de la asignatura Realización del tercer curso de los grados en Comunicación en EADE University of Wales en Málaga como un cambio en la fórmula de trabajo empleada hasta el momento. Este nuevo modelo refuerza la materia teórica relacionada con la historia del cine a través de textos teatrales, elaborados exprofeso o seleccionados por el docente, que el alumnado trabaja en el aula. Estableciendo una dinámica de role playing, el alumno dirige, interpreta y realiza audiovisualmente obras teatrales protagonizadas por personalidades relevantes de la historia del séptimo arte. También se establecen tramas relacionadas con la materia teórica y se incluyen inputs o informaciones relevantes dentro del texto que faciliten la memorización y comprensión de distintos conceptos. 
Esta investigación estudia el modo en que dicha metodología se traslada a la práctica con el objetivo de conocer la utilidad del storytelling y storydoing teatral para la enseñanza-aprendizaje de la historia del cine en las enseñanzas de nivel universitario, así como la opinión del alumnado sobre la implementación de esta práctica.

\section{Marco conceptual}

La implantación del Espacio Europeo de Educación Superior (EEES) conllevó la reformulación de la docencia en la enseñanza superior promovida por la Declaración de Bolonia (Unión Europea, 1999) que, entre otros aspectos, enfatiza un cambio de perspectiva. Respecto a las competencias y resultados de aprendizaje

se aprecian dos particularidades esenciales de la reforma del EEES, casi inseparables entre sí: el carácter eminentemente práctico de los estudios y el vuelco hacia el ámbito empresarial. El enfoque desde un punto de vista práctico, encaminado a la inserción laboral del alumnado, requiere una reformulación del paradigma tradicional enseñanza-aprendizaje. (Martínez Rodrigo y González Fernández, 2012, p.54)

Este cambio en el modo de abordar la enseñanza ha popularizado metodologías como el flipped classroom (Bishop y Verleger, 2013), la gamificación (Cortizo et al., 2011) o la aplicación de las nuevas tecnologías (Cabero, 1994). Sin embargo, ante el auge de las estrategias que otorgan un fuerte protagonismo a la intermediación técnica y tecnológica, es necesario encontrar el equilibrio con las prácticas que no las implementan pero tienen un sólido fundamento pedagógico (Ovelar, Benito y Romo, 2009).

En un contexto en el que la cultura participativa impregna los modos de comunicar y comunicarse en la red, los comportamientos colectivos mediante el social swarming permiten dar respuesta a cuestiones y problemas complejos de forma descentralizada superando las limitaciones de las propuestas individuales (Moya y Moya, 2018). Este principio, extendido entre los más jóvenes debido al uso de las redes sociales y las plataformas colaborativas, es aplicable también a los procesos de aprendizaje formal. 


\subsection{El storytelling en el proceso enseñanza-aprendizaje}

Es interesante observar cómo Green (2004) pone el foco en la capacidad de las historias para ejemplificar conceptos abstractos o complejos, haciendo que la experiencia al explicar dichos conceptos sea más amplia que su simple mención. En una línea similar trabajan Lugmayr, Sutinen, Suhonen, Sedano, Hlavacs y Montero cuando tratan cómo mediante el uso de la narratividad es más sencillo llegar a emocionar al lector o espectador, "an engaged audience that interacts with the narrative flow of a story perceives the narrative as an emotional experience" (Lugmayr et al., 2017, p.15710).

Por su parte Canning, Reinsborough y Smucker (2017) destacan el poder del storytelling para el cambio social y Salmon (2008) plantea sus posibilidades como elemento esclarecedor de la experiencia de la humanidad, aunque también advierte que la política lleva muchos años usando estrategias de storytelling para configurar la visión del mundo que le resulta de su interés. Y es que el concepto se puede definir como la utilización de las historias con un fin concreto, de forma que se da un uso instrumental a la narración para conseguir unos objetivos determinados (Vizcaíno-Alcantud, 2017).

Las posibilidades del storytelling aplicado al aula son numerosas y han sido estudiadas por diversos autores tanto en el ámbito internacional (Abrahamson, 1998; Coulter, Michael y Poynor, 2007) como en el nacional (Illera y Monroy, 2009; Martínez y Pérez del Moral, 2013; Martínez, Pérez del Moral y Piñeiro, 2017). Muchos de estos estudios (Robin, 2008; Lambert, 2013) se centran en el enfoque digital de esta estrategia, por ejemplo, mediante la creación en clase de narraciones usando fotografías (Sadik, 2008), vídeos (Ohler, 2006) o blogs (Abdel-Hack y Helwa, 2014), y especialmente en el ámbito universitario y de educación adulta (Yuksel, Robin y McNeil, 2011); esto es debido a que las TIC presentan numerosas facilidades para interactuar con el alumnado, evaluarlo e implicarlo en la materia (Dreon, Kerper y Landis, 2011).

El storytelling aplicado al aula puede ser muy útil para distintas asignaturas, porque, tal y como defiende Martín González, uno de los condicionantes para que 
su práctica sea eficaz es que la historia que narra "se pueda interpretar como verdadera, que se base en hechos o referencias conocidas y que permita a sus públicos identificarse con ella" (Martín González, 2009, p.12). Esto entronca directamente con las posibilidades del storytelling para el estudio de acontecimientos históricos; de hecho, Davies (2007, p.56) ya menciona que el storytelling "can be used to complement a variety of subjects. The obvious choice might be history and examining how things were in the past through sharing stories".

En el contexto publicitario el storytelling transmite los beneficios de las marcas mediante estrategias narrativas, pero ha surgido una tendencia que promueve una evolución del concepto hacia el storydoing. El storydoing no niega el storytelling sino que amplía su intencionalidad al basarse en que no es suficiente con comunicar, sino que es necesario contar con demostraciones efectivas de las propuestas de valor de las marcas, que deben ser protagonistas de dichas propuestas y no únicamente establecer una narrativa sobre ellas (Luque de Marcos y Baraybar, 2018). Es por ello que storytelling y storydoing son técnicas que pueden complementarse para obtener un mayor impacto sobre el público: las historias que se narran han de ser respaldadas por acciones coherentes con las mismas. En este sentido el storymaking es otro término cercano, ya que se vincula a la construcción y desarrollo de historias como herramientas terapéuticas y educativas (Crimmens, 2006). Si el storytelling consiste en establecer una narrativa con un objetivo determinado y el storydoing en que la historia que se cuenta sea coherente con el comportamiento de quien la instrumentaliza, para que ésta no quede en una simple narración, el storymaking aporta el matiz de construir y desarrollar la historia asumiendo un rol activo en ella.

\subsection{Educación y teatro}

El teatro en la escuela "puede revelarse como un instrumento educativo poderoso" (Oliveira y Stoltz, 2010, p.91) ya que es un recurso motivacional cuyo potencial didáctico viene a apoyar el proceso educativo (Fernández, 2011). A ello se debe que la función del teatro como herramienta divulgadora y facilitadora de la transmisión de conocimientos tenga un amplio recorrido histórico que se inicia en la Antigüedad y que perdura hasta nuestros días. En la Edad Moderna el teatro, 
empleado en parte con objetivos educativos y moralizantes vinculados a la religión tanto en Europa como en América (Viveros, 1992), se constituyó como instrumento curricular para la formación de los jóvenes y se desarrolló el teatro escolar heredando la práctica de los espectáculos medievales que contaban con estudiantes para su realización (Alonso, 2010). También los jesuitas otorgaron gran relevancia al teatro escolar en Europa, América y Asia y distintos pensadores defendieron su utilidad, como es el caso de Jovellanos que abogó por la inclusión del teatro en la educación con fines docentes (Cutillas, 2015).

Ya en el siglo XX los planteamientos de figuras como Piaget, en cuanto a la importancia que otorgó a los juegos dramáticos en el desarrollo durante la infancia (0sorio, 2014), o Dewey, que influyó en la experimentación con juegos, dramatización libre o el aprendizaje activo a través de técnicas de role playing (Bareicha, 2011), potenciaron el uso del teatro como metodología educativa.

En España, en el contexto del aprendizaje informal, el empleo del teatro como mecanismo de transmisión de conocimiento y valores contó con ejemplos tan ilustrativos como las famosas Misiones Pedagógicas durante la Segunda República. En ellas grupos como La Barraca, compuesto mayoritariamente por voluntarios universitarios y dirigido por Federico García Lorca, o el Teatro del pueblo, dirigido por Alejandro Casona, llevaban sus montajes a lo largo de la geografía nacional, principalmente a pueblos pequeños carentes de espacios formales para la representación teatral (Katona, 2014).

Siguiendo a Vieites (2014) existen tres grandes áreas de trabajo en la educación teatral en razón de las finalidades del proceso: educación para el teatro, educación en el teatro y educación por el teatro. Educar para el teatro implica que la formación está orientada al ejercicio profesional de las artes escénicas. Educar en el teatro enmarca el teatro escolar en el sentido en que implica diferentes procesos expresivos que conforman un marco donde desarrollar otras actividades no vinculadas al teatro, así como usos socieducativos o culturales que se desarrollan principalmente en la dimensión no formal. Por último, la educación por el teatro se refiere a acciones formativas vinculadas a otras áreas diferentes del teatro pero que lo emplean como herramienta al servicio de actividades o procesos de aprendizaje 
con un fin propio, por lo que pueden extenderse a ámbitos educativos formales o no formales. En este último caso el teatro se configura como un método aplicable a la educación, pero concebido como un recurso procedimental que se complementa con otros desarrollados de forma conjunta. Cabe recordar que en cualquiera de estas áreas de educación teatral es necesario fijar los roles que desempeñarán los agentes de esa educación, configurando los papeles tanto de profesorado como de alumnado.

Los beneficios del uso del teatro como herramienta educativa son múltiples, ya que no sólo contribuye a fijar conocimientos y vehicula el proceso enseñanzaaprendizaje, sino que permite desarrollar una serie de habilidades y destrezas a través de elementos lúdicos que mejoran la interacción personal, las formas de expresión, de comunicación y el crecimiento personal (Osorio, 2014).

Drama is an influential and beneficial teaching strategy that can be utilized in many ways in the contemporary classroom to provide active, constructivist learning. The history and development of drama as a teaching strategy is a result of valid use in various military, government and corporate business interests throughout the world. (...) drama does engage multi-dimensional learning styles including verbal-linguistic, interpersonal, intrapersonal, kinesthetic, spatial, and logical and often incorporates music, or the music of language. In addition, drama has the ability to enhance reflection in students and can be used to create powerful social learning environments where students develop improvisational speaking and emotional intelligence awareness skills. Drama is an appealing teaching strategy which promotes cooperation, collaboration, self-control, goal-oriented learning as well as emotional intelligence skills. Drama is easily adaptable to a variety of text studies as demonstrated. (Ashton-Hay, 2005, pp.14-15)

El teatro se ha revelado como una herramienta útil en diversos tipos y enfoques de enseñanza donde ha obtenido resultados positivos, como el aprendizaje de idiomas (Miccoli, 2003; Winston, 2013), diferentes programas universitarios (Álvarez y Martín, 2016; Brennan y Pearce, 2009) o el trabajo con alumnos con capacidades especiales (De la Cruz, Lian y Morreau, 1998). 
Además, ya que la educación en valores es posible a través del cine y las artes (López, 2002), esa utilidad se hace extensiva a formas como el teatro. Y es que no sólo "es posible aprender a través del teatro" (Jackson, 2002, p.34) sino que los beneficios a largo plazo de emplear la dramatización en la educación mejoran también las capacidades y habilidades del alumnado para enfrentarse al mundo y al mercado laboral (Pinciotti, 1993; Boudreault, 2010). Por su parte, Courtney (1990) plantea que el aprendizaje consiste en una transformación en el proceso de pensamiento imaginativo que se externaliza a través de la acción dramática.

\section{Metodología}

Esta investigación se circunscribe al análisis de una actividad o experiencia docente desarrollada en la asignatura de Realización del tercer curso de los grados en Publicidad y Relaciones Públicas y en Periodismo de EADE Estudios Universitarios, sede en Málaga de la Universidad de Gales.

La materia, en la que se imparte el módulo Historia del Cine, tiene un peso de 20 créditos ECTS. Desde el curso 2017/2018 se ha implementado en el bloque que aborda la historia del cine una nueva metodología que amplía el modelo de clase magistral para centrarse en un método mixto donde además de explicaciones con un enfoque eminentemente práctico por parte del docente se ha incorporado el storytelling como herramienta de enseñanza-aprendizaje. Se trata de un ejercicio de representación de diversas piezas teatrales con el objetivo de adquirir conocimientos acerca de la historia del cine y es un trabajo con peso específico dentro de la evaluación, ya que supone el $20 \%$ de la nota final.

Las capacitaciones que el alumnado debe adquirir en esta asignatura quedan definidas en su sistema de evaluación, por lo que el profesor conceptualiza este sistema atendiendo a unos resultados de aprendizaje deseados, es decir, los conocimientos que el alumnado debería demostrar para poder superar este apartado de la materia. Según la guía didáctica de la asignatura estos conocimientos son los que siguen: 
- Conocer los diferentes movimientos cinematográficos en la historia del cine.

- Adquirir conocimientos básicos acerca de la producción audiovisual.

- Aplicar estos conocimientos a un desarrollo práctico.

El objetivo de esta investigación sobre la metodología docente aplicada es conocer si las herramientas de storytelling y storydoing teatral se muestran útiles en el aprendizaje de la historia del cine en el ámbito universitario. Como objetivos secundarios se plantean los siguientes:

- OS1: analizar la percepción del alumnado sobre la implementación de esta nueva metodología.

- OS2: valorar la eficacia de esta experiencia docente para favorecer el estudio de la historia del cine.

Para llevar a cabo la investigación sobre este proyecto de innovación educativa se ha optado por una metodología de estudio basada en el uso de un cuestionario (Cerón, 2006) con preguntas cerradas y abiertas con el fin de conocer la percepción del estudiantado sobre la aplicación de este método educativo. La muestra está formada por todo el alumnado de los Grados en Comunicación de EADE University of Wales en Málaga que durante los cursos 2017/2018 y 2018/2019 han cursado la asignatura Realización siguiendo esta metodología. Se ha contado con dos grupos que forman un total de 22 individuos, 11 por año académico.

Los textos teatrales utilizados en la asignatura son los siguientes:

- La Secretaria (Diaz-Morilla, 2015, inédita).

- El Juicio (Diaz-Morilla, 2016, inédita).

- Buñuel y Dali (Trueba, 2006). 
- Tu voz por dentro (Diaz-Morilla, 2015, inédita).

- Flores para Hynkel. La historia real de Nikola Radosevic (Diaz-Morilla, 2018).

Estas piezas, salvo en un caso, han sido escritas por el profesor que imparte la asignatura, el dramaturgo premiado a nivel nacional Pablo Diaz-Morilla, con el objetivo de visibilizar pasajes desconocidos de la historia del cine. Se trata de las siguientes:

- La Secretaria (2015). Escenifica una conversación entre los Hermanos Lumiére y Alice Guy-Blaché, cineasta francesa pionera del cine narrativo. La unidad temática que se trabaja en esta obra son los inicios del cine, con referencias a la productora Gaumont, el sistema de trabajo de los Hermanos Lumiére y las posibilidades técnicas y de entretenimiento del nuevo arte. El género al que pertenece es la comedia. La pieza pone en valor la figura de esta artista y como objetivo secundario pretende que los alumnos reflexionen acerca de cómo las figuras femeninas han sido ocultadas a lo largo de la historia del cine.

- El Juicio (2016). Representa el interrogatorio ficticio llevado a cabo por un censor del régimen estalinista al cineasta S. M. Eisenstein. La unidad temática que se estudia en la pieza es el cine dialéctico soviético de vanguardia, si bien la obra está ambientada a finales de los años 40, con el fin de tratar como tema secundario la importancia de la censura cultural en los diferentes regímenes totalitarios. Toma declaraciones de la obra Reflexiones de un cineasta (1970), de S. M. Eisenstein, recopiladas por Román Gubern, y que incluye fragmentos del Memorándum de la reunión entre Stalin y Eisenstein, meses antes de la muerte del cineasta, así como su última entrevista, recogida por el profesor de cine Ilya Veissfeld. La obra pertenece al género tragedia.

- Buñuel y Dalí (2006). Enclavado en la unidad didáctica de Cine surrealista, en concreto en el análisis de las películas Un perro andaluz (1929) y La edad de oro (1930), este texto es una recopilación de declaraciones y escritos en 
forma de diálogo entre Luis Buñuel y Salvador Dalí realizada por el director y guionista Fernando Trueba en su libro Mi diccionario de Cine (2006). Es la única obra que no pertenece a Diaz-Morilla y corresponde al género teatro documental, al incluir declaraciones reales de ambos artistas sin apenas modificaciones con respecto a su publicación en revistas y periódicos de la época.

- Tu voz por dentro (2015). La unidad temática que comprende es Inicio del cine sonoro y aborda un encuentro imaginario entre el director Lee De Forest y la actriz y cantante Concha Piquer como protagonistas de la primera filmación sonora, fechada en 1923 y anterior a El cantor de Jazz (1927) pero descubierta en el año 2010. Pertenece al género comedia romántica.

- Flores para Hynkel. La historia real de Nikola Radosevic (2018). Comprende la unidad didáctica sobre Charles Chaplin y muestra el interrogatorio de un alto mando del ejército nazi al proyeccionista de cine Nikola Radosevic, que durante la Segunda Guerra Mundial proyectó El Gran Dictador (1940) a un grupo de soldados alemanes. Pertenece al género dramático con toques de comedia.

Todos los personajes empleados (11) son personalidades relevantes a nivel histórico, excepto en dos casos en los que se han utilizado personajes arquetípicos, que son la figura de un censor y la de un general nazi para representar la represión de los regímenes totalitarios.

De estos 9 personajes reales empleados, 5 fueron directores de cine, una de ellas actriz, otro de ellos técnico-inventor, otro un proyeccionista de cine y otro el artista Salvador Dalí, si bien en la obra figura principalmente como coguionista de Un perro andaluz (1929) y guionista de las primeras versiones de La edad de oro (1930).

Los textos empleados en esta metodología están diseñados con el objetivo de vehicular el aprendizaje significativo y reforzar aspectos de la asignatura como promover la capacidad crítica del alumnado, reivindicar el papel de las mujeres 
creadoras, recordar la importancia de la censura en ciertas épocas o conocer declaraciones reales de personajes históricos, al tiempo que la complementan ampliando la visión que ofrece sobre la parte menos conocida de la historia del cine.

Las diferentes piezas de teatro corto han sido seleccionadas con el objetivo de reforzar algunas unidades temáticas de la materia a estudiar. A través de ellas se exploran diferentes aspectos de la historia del cine y se ponen en práctica conocimientos y habilidades adquiridas a lo largo de la asignatura y de forma transversal. El modo en que los alumnos han de trabajar las piezas se establece conforme al siguiente esquema:

1. El profesor plantea los textos con los que se va a trabajar y se sortean entre los estudiantes tanto los papeles como los puestos técnicos que han de ocupar en las distintas obras.

2. De manera individual el alumnado lee cada texto subrayando las referencias a personajes, obras o hechos históricos relevantes.

3. El profesor explica el contenido teórico relacionado con el tema en el que se enmarca la obra teatral que se vaya a trabajar.

4. Los estudiantes que ocupan el rol de director o directora plantean una propuesta de dirección y unas pautas de interpretación, así como una planificación audiovisual para la fase de grabación de la obra. El profesor ejerce una labor de guía durante este proceso.

5. En grupo con ayuda del profesor ensayan la pieza para posteriormente representarla en el aula y que cada uno de ellos ejecute sus funciones de manera coordinada.

6. Tras el montaje se realiza un debate y una revisión crítica del proceso por parte de todo el equipo, donde el profesor aporta notas técnicas sobre el ejercicio. 
7. Al finalizar la interpretación y grabación de las diferentes piezas los alumnos realizan por grupos una edición del metraje obtenido en la grabación teatral a dos cámaras.

8. Como fase final se realiza el visionado de todas las piezas, el profesor aporta correcciones técnicas y se ponen en común las conclusiones de los valores y el refuerzo sobre el contenido teórico de la materia aportados por los proyectos, así como sobre el proceso de aprendizaje y las habilidades desarrolladas a través de esta actividad.

Durante este proceso se ponen en práctica conocimientos y habilidades específicas y transversales adquiridas previamente, como son conceptos propios de planificación y realización audiovisual, solución de problemas y dinámicas de comunicación en público, así como trabajo en equipo.

Este proceso de trabajo colaborativo hace que los alumnos se conviertan en protagonistas del aprendizaje, dejando atrás un rol pasivo para adoptar un papel activo que posibilite el desarrollo de la clase y que ponga el foco sobre ellos en lugar de que permanezca de forma exclusiva sobre el docente.

\section{Resultados y discusión}

El análisis de los cuestionarios ha arrojado unos resultados muy positivos en líneas generales, ya que la satisfacción con la metodología es alta debido a que desde el punto de vista del estudiantado contribuye al aprendizaje significativo.

A la pregunta '¿Qué te ha parecido el enfoque de la asignatura? (emplear role play vinculándola con el teatro)' el 72,7\% de los alumnos del 2017/2018 tenían una respuesta positiva y el $81,8 \%$ de los de 2018/2019. En ambos cursos un $72,7 \%$ del alumnado opinó que la metodología a desarrollar era sencilla de comprender y un $27,3 \%$ de cada año que era medianamente sencilla; ningún estudiante indicó que le hubiera resultado difícil de asimilar. 
Respecto a la aplicación de esta metodología a otras asignaturas el 72,7\% y el $54,5 \%$ opinaron en $2017 / 2018$ y en $2018 / 2019$ respectivamente que debería aplicarse en otras materias; el $18,2 \%$ y el $45,5 \%$ respectivamente creen que tal vez debiera aplicarse.

El alumnado destaca de esta metodología ventajas como el enfoque práctico ( $63,6 \%$ en $2017 / 2018$ y $81,8 \%$ en $2018 / 2019$ ), el enfoque participativo ( $36,4 \%$ en $2017 / 2018$ y $81,8 \%$ en $2018 / 2019$ ), el aprender desde otro punto de vista diferente al habitual (63,6\% en $2017 / 2018$ y $72,7 \%$ en $2018 / 2019)$, facilidad para recordar (63,6\% en $2017 / 2018$ y $54,5 \%$ en $2018 / 2019)$ y el enfoque divertido de la materia (54,5\% en $2017 / 2018$ y $36,4 \%$ en $2018 / 2019)$. Ningún alumno ha indicado que no destacaría nada de lo aprendido en la asignatura.

Respecto a las preguntas sobre si al alumnado le gustan las asignaturas vinculadas a la historia y si le gusta en concreto la asignatura de Historia del Cine, en el segundo caso el $95 \%$ de las respuestas son positivas (tabla 1) por lo que la diferencia resulta sustancial.

\begin{tabular}{|c|c|c|c|c|}
\hline \multicolumn{4}{|c|}{ Comparativa pregunta sobre afinidad con asignaturas vinculadas a } \\
Historia y la asignatura Historia del Cine \\
\hline & \multicolumn{2}{|c|}{ Asignaturas de Historia } & \multicolumn{2}{c|}{ Historia del Cine } \\
& & & & \\
\hline Respuestas & $\mathbf{2 0 1 7 / 2 0 1 8}$ & $\mathbf{2 0 1 8 / 2 0 1 9}$ & $\mathbf{2 0 1 7 / 2 0 1 8}$ & $\mathbf{2 0 1 8 / 2 0 1 9}$ \\
\hline Positivas & $36.4 \%$ & $81.9 \%$ & $90.9 \%$ & $100 \%$ \\
\hline Neutras & $9.1 \%$ & $9.1 \%$ & - & - \\
\hline Negativas & $54.6 \%$ & $9.1 \%$ & $9.1 \%$ & - \\
\hline
\end{tabular}

Tabla 1: Comparativa sobre afinidad con asignaturas vinculadas a Historia y la asignatura Historia del Cine.

Elaboración propia. 
Frente a la pregunta ¿¿Qué has aprendido durante el proceso de la asignatura?' las respuestas son variadas pero la mayoría coinciden en resaltar que el aprendizaje se puede realizar desde una perspectiva diferente, más atractiva, que permite asimilar mejor el conocimiento (tabla 2): “Mediante la práctica del teatro es mucho más fácil aprender la historia, y no solo eso, sino que, además, es fácil recordar anécdotas y datos que de manera teórica es más aburrido y complejo"; "La historia desde otro punto de vista, ponerte en el lugar del protagonista. Aprender jugando".

\section{Respuestas a la pregunta de respuesta libre sobre aprendizaje en la asignatura}

Mejorar la capacidad de oratoria y perder el miedo escénico.

Es mas fácil aprender desde un punto de vista practico porque me florece más fácilmente el interés.

La información se te queda antes.

He aprendido lo importante que han sido los aportes de los cineastas para el desarrollo de la comunicación audiovisual, además he comprendido el esfuerzo tan necesario que supone trabajar en equipo.

Expresar tus pensamientos en público.

A visualizar el cine desde distintas perspectivas.

A trabajar en equipo.

Una parte de la historia del cine que desconocía y que me ha resultado muy interesante, a la vez que investigaba y descubría personalidades de este campo.

Cómo se expresaban y su historia.

La trayectoria de muchos directores de cine.

Un nuevo método para recordar Historia del Cine.

Cómo eran las personas que estudiamos en la asignatura y más información sobre su vida.

Que mediante la práctica del teatro es mucho más fácil aprender la historia, y no solo eso, sino que, además, es fácil recordar anécdotas y datos que de manera teórica es más aburrido y complejo. 


\begin{tabular}{|l|}
\hline \multicolumn{1}{|c|}{$\begin{array}{c}\text { Respuestas a la pregunta de respuesta libre sobre aprendizaje } \\
\text { en la asignatura }\end{array}$} \\
\hline Que la historia puede enfocarse desde puntos de vista que no conocíamos con anterioridad. \\
\hline Entender el cine en la actualidad, el proceso llevado a cabo para llegar al cine que vemos ahora. \\
\hline $\begin{array}{l}\text { La historia sobre determinados personajes relevantes del ámbito audiovisual y su contexto his- } \\
\text { tórico, así como la personalidad de los mismos reflejada en los diálogos. }\end{array}$ \\
\hline Apreciar mucho más todo lo relacionado con el teatro y con Historia de Cine. \\
\hline La historia desde otro punto de vista, ponerte en el lugar del protagonista. "Aprender jugando". \\
\hline Muchas cosas como el enfoque de planos, técnicas, fallos de raccord... \\
\hline Pensaba que iba a aprender menos y más despacio. \\
\hline No creía que hubiese clases tan prácticas. \\
\hline Mis expectativas han sido superadas con creces. \\
\hline Esperaba que fuera una asignatura tediosa y me ha resultado divertida. \\
\hline Creía que iba a estudiar largo y tendido sobre historia pero me ha sorprendido positivamente. \\
\hline
\end{tabular}

Tabla 2: Respuestas a la pregunta sobre aprendizaje en la asignatura. Elaboración propia.

La pregunta ¿¿Qué destacarías sobre las prácticas?' también es abierta para que los alumnos se expresen con libertad. Las respuestas realzan el carácter divertido del aprendizaje, que les facilita alcanzar nuevos conocimientos despertando su interés y desarrollando habilidades como hablar en público, trabajar en equipo o aplicar los conocimientos adquiridos (tabla 3). Algunas respuestas en este sentido son: "Me parece divertida y una forma complementaria que puede servirnos mucho para saber más de la asignatura. Además, es bueno para trabajar en equipo y perder miedo escénico lo cual considero aptitudes fundamentales" o "Es una forma de desarrollar el conocimiento adquirido durante las clases de realización y aplicarlos de una forma práctica sobre la obra". 


\section{Respuestas a la pregunta de respuesta libre sobre qué destacarian de las prácticas}

En la práctica, ayuda a interesarse más por la asignatura.

El material multimedia que apoya la teoría.

Es divertida y se disfruta y más cuando te gusta el teatro.

La diversión ha sido uno de los factores que más ha impulsado las ganas de realizar la práctica, también cabe destacar la importancia de las relaciones entre compañeros a nivel comunicativo.

La mejora de relación con los compañeros.

Aprender de una manera más práctica, una asignatura que es teórica.

El dinamismo y lo divertido.

Destacaría que, casi sin darte cuenta, te aprendes la historia del cine a través del ensayo del teatro.

Muchas de ellas eran divertidas e interesantes.

El descubrir directores que me gustan.

La labor de dirección y caracterización.

Aprendemos historia del cine, dinámicas de cada director...

Aprendo dinámicas personales e historia del cine.

La forma de ver otro punto de vista de la historia.

Que me parece divertida y una forma complementaria que puede servirnos mucho para saber más de la asignatura. Además, es bueno para trabajar en equipo y perder miedo escénico lo cual considero aptitudes fundamentales.

Los conocimientos de la historia del cine, la censura con la que han tenido que lidiar grandes cineastas y las metodologías que utilizan para poner en práctica el cine.

Es una forma de desarrollar el conocimiento adquirido durante las clases de realización y aplicarlos de una forma practica sobre la obra.

La forma divertida de entender la asignatura.

La originalidad y las diferencias de cada texto.

Conocimiento, cultura, historia, hablar en público.

Tabla 3: Respuestas a la pregunta sobre qué destacarían de las prácticas. Elaboración propia. 
En la pregunta sobre las habilidades desarrolladas destacan la capacidad para hablar en público, el desarrollo del lenguaje no verbal y el trabajo en equipo (gráfico 1).

\section{Habilidades destacadas por el alumnado}

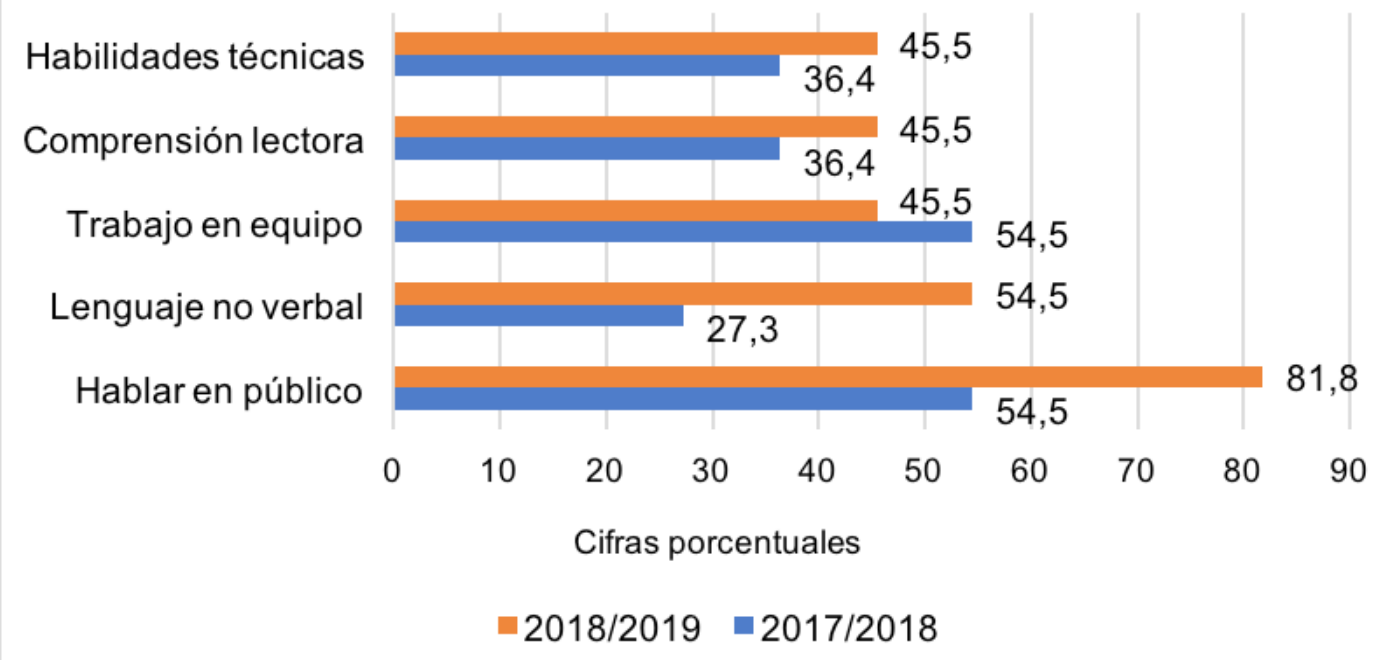

Gráfico 1: Habilidades destacadas por el alumnado.

Elaboración propia.

Las opiniones sobre la inclusión del storytelling y el storydoing como parte de la metodología son ampliamente positivas: en ambos casos el $81,8 \%$ en el curso $2017 / 2018$ y el $90,9 \%$ en el curso 2018/2019 opinan positivamente sobre el empleo de las mismas.

La mayoría del alumnado responde afirmativamente sobre si esta metodología debe aplicarse de forma complementaria a otras; en 2017/2018 así lo indica el $81,8 \%$, cifra que desciende ligeramente al curso siguiente $(72,7 \%)$, donde el $27,3 \%$ opina que tal vez sea recomendable incorporarla de forma complementaria.

En cuanto a las valoraciones de la asignatura, del rol desempeñado por el profesor y por el estudiante, los resultados son muy positivos (gráfico 2). Cabe destacar 
que no hay respuestas negativas, sino que aquellas que no son positivas o muy positivas se sitúan en valores neutros.

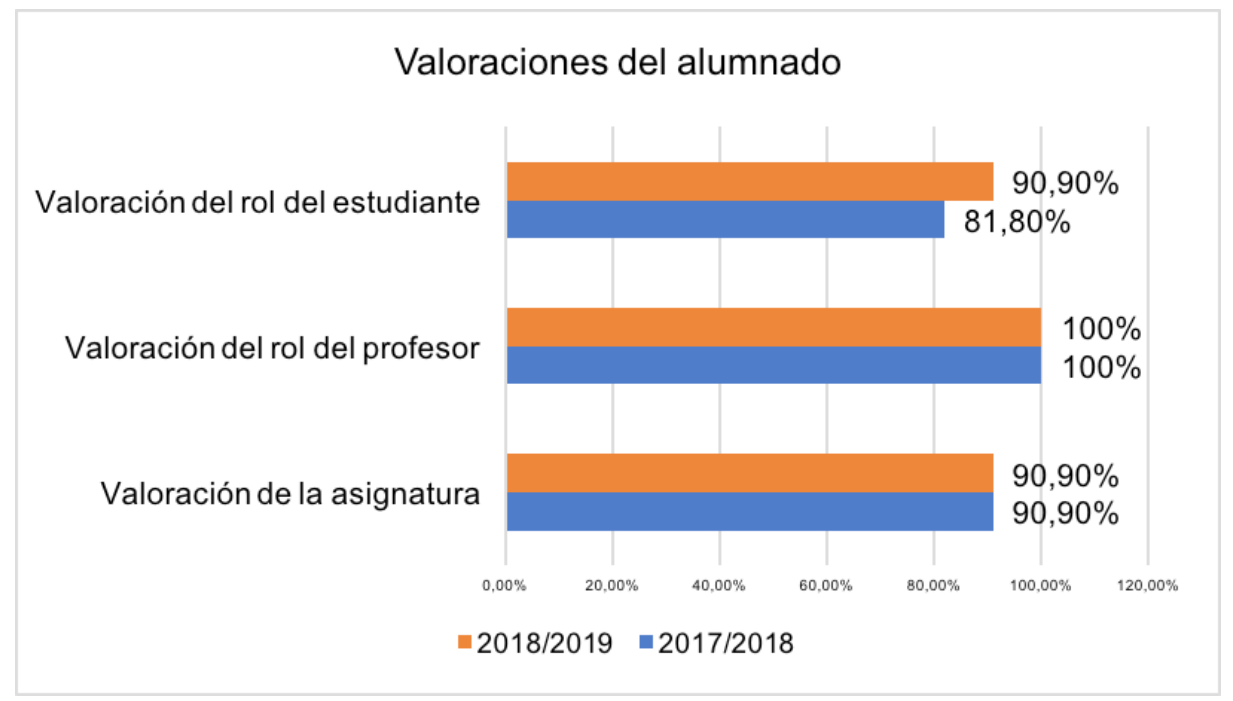

Gráfico 2: Valoraciones del alumnado.

Elaboración propia.

En la última pregunta del cuestionario se pide al alumnado que valore lo aprendido comparativamente con otras materias y los resultados indican mayoritariamente que se aprende más o mucho más que en otras asignaturas (tabla 4). Las respuestas negativas se limitan a dos que valoran que han aprendido poco sobre Responsabilidad/autonomía personal.

\begin{tabular}{|l|c|}
\hline \multicolumn{2}{|c|}{ Respuestas a la pregunta 'Valora el aprendizaje de la asignatura' } \\
\hline & $\begin{array}{c}\text { He aprendido más de lo } \\
\text { habitual/ mucho más de lo } \\
\text { habitual }\end{array}$ \\
\hline Materia propia de la asignatura (en este caso Historia del Cine) & $77.25 \%$ \\
\hline Aspectos técnicos & $54.55 \%$ \\
\hline Responsabilidad/autonomía personal & $59.1 \%$ \\
\hline Trabajo colaborativo & $50 \%$ \\
\hline
\end{tabular}

Tabla 4: Respuestas a la pregunta 'Valora el aprendizaje de la asignatura'. Elaboración propia. 
El análisis indica que se produce una construcción social del conocimiento al aplicar una metodología activa que supera el esquema clásico de transmisión descendente para implementar una estrategia de aprendizaje en la que el alumno se convierte en protagonista. El enfoque participativo se vehicula mediante el storytelling y el storydoing teatral, de forma que la narratividad permite nuevas formas de abordaje del conocimiento sobre Historia del Cine. Esto coincide con lo planteado por Davies (2007) sobre la utilidad de esta técnica aplicada a materias vinculadas a la historia. Por su parte, el storydoing traslada la acción del docente a los estudiantes, que dan forma a los textos teatrales y a su cobertura audiovisual, convirtiéndose en hacedores de historias.

De este modo se reformula el paradigma tradicional de enseñanza transmisiva en busca de un enfoque práctico y que aumente la empleabilidad del alumnado, coincidiendo con los planteamientos ya establecidos por otros autores (Martínez Rodrigo y González Fernández, 2012). Se construye el aprendizaje superando las limitaciones individuales y emulando la construcción de cultura participativa que se da en las redes (Moya y Moya, 2018) pero a través de la aplicación de estrategias analógicas a las que el alumnado está poco acostumbrado debido a la relevancia actual de lo digital (0velar, Benito y Romo, 2009). Los resultados muestran que el teatro es una herramienta apropiada para desarrollar las inteligencias múltiples y habilidades transversales, además de para presentar contenidos de forma amena y atractiva y para educar en valores, en la misma línea de los aportes previos de Courtney (1990), Jackson, (2002) y López (2002) respectivamente. Además, al tiempo se asientan y trabajan aptitudes y actitudes útiles para la vida (Osorio, 2014; Boudreault, 2010).

El papel del docente en este proceso es fundamental y altamente valorado por los estudiantes ya que tiene una doble vertiente: por una parte es el encargado de elaborar y seleccionar las piezas a representar teniendo como condicionante la base histórica de las obras (Martín González, 2009) para que éstas sirvan de apoyo a los contenidos teóricos; por otra parte el papel de acompañante y guía en el aprendizaje, superando el rol de mero transmisor para ayudar al alumnado a plasmar en la práctica los conocimientos adquiridos promoviendo la solución de problemas de forma colaborativa. 


\section{Conclusiones}

A lo largo de los siglos el teatro y las técnicas dramáticas se han empleado como herramientas didácticas aplicables al aprendizaje formal e informal. En el entorno escolar el teatro se configura como un recurso de gran utilidad motivadora y dinamizadora que puede ser aplicado a diferentes áreas con el objetivo de complementar los conocimientos adquiridos por el alumnado y de transformar en colaborativos los espacios y dinámicas individuales de aprendizaje.

Esta investigación profundiza en la aplicación de la filosofía de educación por el teatro o mediante el teatro en el entorno de enseñanzas universitarias. En concreto se enfoca al área de comunicación al centrarse en una nueva fórmula didáctica para transmitir conocimientos sobre la historia del cine a nivel teórico y práctico. Esto se logra gracias a una doble condición: en primer lugar, una selección de obras teatrales que refuercen de manera adecuada los contenidos teóricos; $\mathrm{y}$, en segundo lugar, mediante la aplicación de una metodología activa de aprendizaje significativo que erija al alumnado como protagonista.

Gracias a la selección y elaboración ad hoc de material por parte del docente, especialista tanto en Historia del Cine como en dramaturgia, se logran cimentar aspectos teóricos de la asignatura que suelen resultar de mayor dificultad para los estudiantes.

Por otra parte, se emplean herramientas metodológicas que suponen un refuerzo en el proceso de adquisición de conocimiento a través del desarrollo en el aula de estrategias de storytelling, ya que la narratividad que caracteriza a este recurso aumenta la efectividad nemotécnica. A su vez, la práctica del storydoing y storymaking ayuda a centrar el foco de la acción-aprendizaje en el alumnado, que adopta diferentes roles a través de los que conjuga conocimientos y habilidades propias de la materia con otros contenidos transversales respecto al currículo. En este enfoque el docente supera el papel de transmisor para convertirse en guía y acompañante durante todo el proceso y darle relevancia a su figura, que se conforma como eje dinamizador y motivador. 
En respuesta a los objetivos planteados por esta investigación se concluye que la aplicación del storytelling y el storydoing teatral se muestra como una estrategia de utilidad que favorece el proceso de enseñanza-aprendizaje de la materia Historia del Cine en el ámbito universitario. Asimismo, se presenta como una metodología eficiente y que supone un valor añadido para el estudiantado, que evalúa esta innovación docente de manera muy positiva. Con el empleo de esta metodología de aprendizaje no solo se ha potenciado la adquisición de conocimientos teóricos vinculados a la historia del cine, sino que el alumnado ha desarrollado habilidades que aumentan su empleabilidad, al tiempo que incrementa también sus recursos en otras áreas propias de las inteligencias múltiples.

Las limitaciones de esta investigación se concretan en la propia naturaleza de la aplicación del proyecto de innovación educativa a un grupo reducido de estudiantes. Sin embargo, los buenos resultados obtenidos en la valoración de esta metodología de aprendizaje por parte del alumnado indican la posibilidad de transferencia de la práctica tanto a otros grupos más numerosos como a otras materias que aborden la enseñanza de la historia del cine en diferentes ámbitos.

\section{Referencias bibliográficas}

Abdel-Hack, E. M. \& Helwa, H. S. A. A. (2014). Using digital storytelling and weblogs instruction to enhance EFL narrative writing and critical thinking skills among EFL majors at faculty of education. Educational Research, 5(1), 8-41. doi: http://dx.doi.org/10.14303/er.2014.011

Abrahamson, C. E. (1998). Storytelling as a pedagogical tool in higher education. Education, 118(3), 440-452. Recuperado de https://tinyurl.com/y5kx3lyf Alonso Asenjo, J. (2010). Bases y despegue del teatro como instrumento educativo en la Edad Moderna. TeatrEsco: Revista del Antiguo Teatro Escolar Hispánico, (4), 2-61. Recuperado de https://tinyurl.com/y5e8xatv

Álvarez, P. \& Martín, A. (2016). El teatro como herramienta didáctica en la enseñanza de la Historia de la Educación Contemporánea. Revista Digital de Investigación en Docencia Universitaria, 10(1), 41-51. doi: http://dx.doi. org/10.19083/ridu.10.459 
Ashton-Hay, S. (2005). Drama: Engaging all learning styles. From the Proceedings of 9th INGED (Turkish English Education Association) International Conference 'New Horizons in ELT' Economics and Technology University in Ankara, Turkey, 2005. Recuperado de https://tinyurl.com/y5jxdygb

Bishop, J. L. \& Verleger, M. A. (2013, June). The flipped classroom: A survey of the research. In ASEE national conference proceedings, Atlanta, GA, 30(9), 1-18. Recuperado de https://tinyurl.com/y74c8fa3

Brennan, R. \& Pearce, G. (2009). Educational drama: A tool for promoting marketing learning. International Journal of Management Education, 8(1), 1-10. Recuperado de https://tinyurl.com/yyjzod6g

Boudreault, C. (2010). The benefits of using drama in the ESL/EFL classroom. The Internet TESL Journal, 16(1), 1-5. Recuperado de http://iteslj.org/Articles/Boudreault-Drama Cabero Almenara, J. (1994). Nuevas tecnologías, comunicación y educación. Comunicar: revista científica iberoamericana de comunicación y educación, (03), 14-25. Recuperado de http://hdl.handle.net/10272/689

Canning, D., Reinsborough, P. \& Smucker, J. M. (2017). Re: Imagining change: How to use story-based strategy to win campaigns, build movements, and change the world. Pm Press.

Cerón, M. C. (2006). Metodologías de la investigación social. LOM ediciones. Coulter, C., Michael, C. \& Poynor, L. (2007). Storytelling as pedagogy: An unexpected outcome of narrative inquiry. Curriculum Inquiry, 37(2), 103-122. doi: https:// doi.org/10.1111/j.1467-873X.2007.00375.x

Cortizo Pérez, J. C., Carrero García, F. M., Monsalve Piqueras, B., Velasco Collado, A., Díaz del Dedo, L. I. \& Pérez Martín, J. (2011). Gamificación y Docencia: Lo que la Universidad tiene que aprender de los Videojuegos. Universidad Europea. Recuperado de http://hdl.handle.net/11268/1750

Courtney, R. (1990). Drama and Human Learning. In Courtney, R. Drama and Intelligence: A Cognitive Theory, 138-148. McGill-Queen's University Press.

Crimmens, P. (2006). Drama therapy and storymaking in special education. Jessica Kingsley Publishers.

Cutillas Sánchez, V. (2015). El teatro y la pedagogía en la historia de la educación. Tonos digital, 28(0). Recuperado de https://tinyurl.com/y4uwflfc

Davies, A. (2007). Storytelling in the classroom: Enhancing traditional oral skills for teachers and pupils. Sage. 
De la Cruz, R. E., Lian, M. C. J. \& Morreau, L. E. (1998). The effects of creative drama on social and oral language skills of children with learning disabilities. Youth Theatre Journal, 12(1), 89-95. Recuperado de https://tinyurl.com/y67pvpq9 Diaz-Morilla, P. (2018). Flores para Hynkel. La historia real de Nikola Radosevic. En Campos Suárez, G. (coord.) Incisos y concisos. Teatro 15 textos breves. Málaga: El toro celeste.

Dreon, 0., Kerper, R. M. \& Landis, J. (2011). Digital storytelling: A tool for teaching and learning in the YouTube generation. Middle School Journal, 42(5), 4-10. Recuperado de https://tinyurl.com/y4e4p5zr

Eisenstein, S. M. (1970). Reflexiones de un cineasta. Lumen.

Fernández, J. J. P. (2011). Motivar en Secundaria: el teatro: una herramienta eficaz. Erasmus Ediciones.

Green, M. C. (2004). Storytelling in teaching. APS Observer, 17(4). Recuperado de: https://tinyurl.com/yyuox3jk

Illera, J. L. R. \& Monroy, G. L. (2009). Los relatos digitales y su interés educativo. Educação, Formação \& Tecnologias 2(1), 5-18. Recuperado de: https://tinyurl. com/y3m8blam

Katona, E. (2014). Teatros ambulantes en la Segunda República española. Colindancias: Revista de la Red de Hispanistas de Europa Central, (5), 39-61. Recuperado de https://tinyurl.com/yyams49n

Lambert, J. (2013). Digital storytelling: Capturing lives, creating community. Routledge. doi: https://doi.org/10.4324/9780203102329

López, S. 0. (2002). La educación en valores a través del cine y las artes. Revista iberoamericana de educación, (29), 157-175. doi: https://doi.org/10.35362/ rie290955

Lugmayr, A., Sutinen, E., Suhonen, J., Sedano, C. I., Hlavacs, H. \& Montero, C. S. (2017). Serious storytelling-a first definition and review. Multimedia tools and applications, 76(14), 15707-15733. doi: https://doi.org/10.1007/s11042-016-3865-5

Luque de Marcos, J. \& Baraybar Fernández, A. (2018). Nuevas tendencias en la construcción de marcas: una aproximación al storydoing. Revista Prisma Social, 0(23), 435-458. Recuperado de http://revistaprismasocial.es/article/view/2745 Martín González, J. A. (2009). La eficacia del Storytelling. MK Marketing+ Ventas, (251), 8-17. Recuperado de https://tinyurl.com/y3y4k83q 
Martínez, L. V. \& Pérez, M. E. D. M. (2013). 'Digital storytelling': una nueva estrategia para narrar historias y adquirir competencias por parte de los futuros maestros. Revista Complutense de Educación, 25(1), 115. doi: http://dx.doi. org/10.5209/rev_RCED.2014.v25.n1.41237

Martínez Rodrigo, E. \& González Fernández, A. (2012). Renovación, innovación y TIC en el EEES. Revista ICON014 Revista Científica De Comunicación Y Tecnologías Emergentes, 7(3), 50-63. doi: https://doi.org/10.7195/ri14.v7i3.302

Miccoli, L. (2003). English through drama for oral skills development. ELT journal, 57(2), 122-129. Recuperado de https://tinyurl.com/yxovj9lp

Moya E. \& Moya, J. (2018). Prosumo, Swarming y Transmedia. Hacia un nuevo concepto de Stakeholder. Revista ICON014 Revista Científica De Comunicación Y Tecnologías Emergentes, 16(2), 25-50. doi: https://doi.org/10.7195/ri14. v16i2.1213

Ohler, J. (2006). The world of digital storytelling. Educational leadership, 63(4), 44-47. Recuperado de https://www.learntechlib.org/p/98782/

Oliveira, M. \& Stoltz, T. (2010). Teatro na escola: considerações a partir de Vygotsky. Educar em Revista, 26(36), 77-93. doi: http://dx.doi.org/10.1590/S010440602010000100007

Osorio, A. (2014). El teatro va a la escuela. Organización de Estados Iberoamericanos para la Educación, la Ciencia y la Cultura. Madrid.

Ovelar Beltrán, R., Benito Gómez, M. \& Romo Uriarte, J. (2009). Nativos digitales y aprendizaje. Revista ICON014 Revista Científica De Comunicación Y Tecnologías Emergentes, 7(1), 31-53. doi: https://doi.org/10.7195/ri14.v7i1.332

Pérez, M. E. D. M., Martínez, L. V. \& Piñeiro, M. R. N. (2017). Competencias comunicativas y digitales impulsadas en escuelas rurales elaborando digital storytelling. Aula Abierta, 45(1), 15-24. doi: http://dx.doi.org/10.17811/ rifie.45.2017.15-24

Pinciotti, P. (1993). Creative Drama and Young Children: The Dramatic Learning Connection, Arts Education Policy Review, 6(94), 24-28. doi: 10.1080/10632913.1993.9936938

Robin, B. R. (2008). Digital storytelling: A powerful technology tool for the 21st century classroom. Theory into practice, 47(3), 220-228. doi: https://doi. org/10.1080/00405840802153916 
Sadik, A. (2008). Digital storytelling: A meaningful technology-integrated approach for engaged student learning. Educational technology research and development, 56(4), 487-506. doi: https://doi.org/10.1007/s11423-008-9091-8

Salmon, C. (2008). Storytelling: la máquina de fabricar historias y formatear las mentes. Barcelona: Península.

Trueba, F. (2006). Mi diccionario de cine. Barcelona: Galaxia Gutenberg.

Unión Europea. (1999). Declaración de Bolonia. Declaración conjunta de los ministros europeos de educación. Bolonia. Recuperado de http://www.eees.es/ pdf/Bolonia_ES.pdf

Vieites, M. F. (2014). La educación teatral: nuevos caminos en historia de la educación. Historia de la educación, (33), 325-350. Recuperado de https:// tinyurl.com/yy4qph6z

Viveros, G. (1992). El teatro como instrumento educativo en el México del siglo XVIII. Estudios de historia novohispana, 12(012). Recuperado de http:// revistas.unam.mx/index.php/ehn/article/view/3349

Vizcaíno Alcantud, P. J. (2017). Del storytelling al storytelling publicitario: el papel de las marcas como contadoras de historias (Tesis doctoral). Universidad Carlos III de Madrid, Madrid. Recuperado de http://hdl.handle.net/10016/24104

Winston, J. (Ed.). (2013). Second language learning through drama: Practical techniques and applications. Routledge.

Yuksel, P., Robin, B. \& McNeil, S. (2011, March). Educational uses of digital storytelling all around the world. In Society for Information Technology \& Teacher Education International Conference, 1264-1271. Association for the Advancement of Computing in Education (AACE). Recuperado de https://tinyurl.com/y22uovw8

\section{Filmografía}

Buñuel, L. (1929). Un perro andaluz. Francia.

Buñuel, L. (1930). La Edad de Oro. Francia: Vicomte de Noailles.

Chaplin, C. (1940). El Gran Dictador. EEUU: United Artists.

Crosland, A. (1927). El cantor de Jazz. EEUU: Warner Bros.

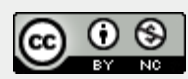

Esta obra está bajo una licencia de Creative Commons Reconocimiento-NoComercial 4.0 Internacional. 